\title{
TRIP13 is expressed in colorectal cancer and promotes cancer cell invasion
}

\author{
KENJI KURITA ${ }^{1}$, MASAO MAEDA ${ }^{2}$, MOHAMMED A. MANSOUR ${ }^{2,3}$, TOSHIO KOKURYO ${ }^{1}$, KEISUKE UEHARA ${ }^{1}$, \\ YUKIHIRO YOKOYAMA ${ }^{1}$, MASATO NAGINO ${ }^{1}$, MICHINARI HAMAGUCHI ${ }^{2}$ and TAKESHI SENGA ${ }^{2}$ \\ ${ }^{1}$ Department of Surgical Oncology; ${ }^{2}$ Division of Cancer Biology, Nagoya University Graduate School of Medicine, Nagoya \\ 466-8550, Japan; ${ }^{3}$ Biochemistry Section, Department of Chemistry, Faculty of Science, Tanta University, Tanta 31527, Egypt
}

Received June 27, 2015; Accepted August 19, 2016

DOI: $10.3892 / \mathrm{ol} .2016 .5332$

\begin{abstract}
Thyroid hormone receptor interactor 13 (TRIP13) is a member of the ATPases associated with various cellular activities family of proteins and is highly conserved in a wide range of species. Recent studies have demonstrated that TRIP13 is critical for the inactivation of the spindle assembly checkpoint and is associated with the progression of certain cancers. In the present study, the role of TRIP13 in colorectal cancer (CRC) was examined. Reverse transcription-quantitative polymerase chain reaction analysis revealed that TRIP13 messenger RNA was highly expressed in multiple CRC tissues. The depletion of TRIP13 in CRC cells suppressed cell proliferation, migration and invasion. To determine whether the catalytic activity of TRIP13 was critical for cancer progression, an inactive mutant of TRIP13 was expressed in CRC cells. The invasion of cancer cells that expressed the mutant TRIP13 was significantly reduced compared with that of the wild type TRIP13-expressing cancer cells. These results indicate that TRIP13 could be a potential target for CRC treatment.
\end{abstract}

\section{Introduction}

The ATPases associated with various cellular activities (AAA+) family of proteins comprises a functionally different group of enzymes that are involved in an array of cellular processes, including protein degradation, protein-complex disassembly and DNA replication (1). The AAA+ proteins are present in all kingdoms, and are characterized by the presence of conserved AAA+ domains that contain Walker A and Walker B motifs, followed by a conserved region called second region of homology (2). The majority of AAA+

Correspondence to: Dr Takeshi Senga, Division of Cancer Biology, Nagoya University Graduate School of Medicine, 65 Tsurumai, Showa, Nagoya 466-8550, Japan

E-mail: tsenga@med.nagoya-u.ac.jp

Key words: TRIP13, invasion, migration, AAA+, colorectal cancer proteins form hexamers to exert their biological functions. The chemical energy generated by adenosine triphosphate (ATP) hydrolysis by this family of proteins induces conformational changes in the substrate proteins to modulate their functions $(3,4)$.

A previous study reported that certain AAA+ enzymes are associated with tumor progression (5). For example, reptin [also known as RuvB-like (RUVBL) 2] and pontin (RUVBL1) have been demonstrated to be overexpressed in several tumors (5). These proteins interact with c-Myc or $\beta$-catenin, thus modulating their transcriptional activities to promote tumor progression (6). AAAt proteins hydrolyze ATP, and thus, chemical inhibitors that disrupt the activities of cancer-associated AAA+ proteins may represent promising anti-cancer drugs.

Thyroid hormone receptor interactor 13 (TRIP13, also known as 16E1BP and pachytene checkpoint 2) is a member of the AAA+ family proteins and is conserved in a wide range of species (7). It was first identified as a protein that interacts with human papilloma virus E1 proteins by a yeast two-hybrid analysis, but the physiological function of the interaction remains unknown (7). Accumulating studies have demonstrated that TRIP13 plays pivotal roles in meiotic recombination and DNA repair in plants, yeast, worms and mice (8-12). TRIP13 forms a stable hexameric ring, and ATP binding, as well as ATP hydrolysis, are critical for the function of the protein (13). Previous studies have revealed that TRIP13 is a novel component of the spindle assembly checkpoint (SAC) pathway (14-17), which is crucial for the accurate distribution of duplicated chromosomes (18). Defects in the SAC pathway induce failure in chromosome separation and result in aneuploidy, which eventually leads to cellular apoptosis or transformation (19). TRIP13 was also reported to promote nonhomologous end-joining, and its overexpression resulted in cellular transformation and resistance to chemotherapeutic agents, indicating that aberrant expression of TRIP13 may be associated with tumor progression (20).

The present study examined whether TRIP13 has a tumor-promoting function in colorectal cancer (CRC) using human CRC tissue samples and cell lines. The results demonstrated that TRIP13 is highly expressed in CRC tissues and that its depletion suppresses the malignant characteristics of CRC cells. 


\section{Materials and methods}

Cells and antibodies. HCT116 and DLD1 cells were purchased from the American Type Culture Collection (Manassas, VA, USA) and were cultured in Dulbecco's modified Eagle medium (DMEM; Wako Pure Chemical Industries, Ltd., Osaka, Japan) and RPMI 1640 medium (Wako Pure Chemical Industries, Ltd.), respectively, supplemented with $10 \%$ fetal bovine serum (FBS; Biowest, Nuaille, France) and antibiotics. Cells were authenticated by short tandem repeat analysis using GenePrint $^{\circledR} 10$ System (Promega Corporation, Madison, WI, USA) in 2014. HEK293T cells (RIKEN BioResource Center, Tsukuba, Japan) were used to produce recombinant retroviruses and were maintained in DMEM with $10 \%$ FBS. The anti-TRIP13 antibody (cat. no. A303-605A) was obtained from Bethyl Laboratories (Montgomery, TX, USA) and the anti-Flag antibody (cat. no. 1E6) from Wako Pure Chemical Industries, Ltd. Cells were incubated with the antibodies for $1 \mathrm{~h}$ at room temperature for immunoblot analysis.

Generation of stable cell lines. Full-length TRIP13 RNA was extracted from HCT116 cells using the RNeasy Mini Kit (Qiagen, Venlo, The Netherlands). RNA was amplified by polymerase chain reaction (PCR) following reverse-transcription using PrimeScript Reverse Transcriptase (Takara Bio, Inc., Otsu Japan) and was cloned into the pQCXIP retroviral vector (Clontech Laboratories, Inc., Mountainview, CA, USA) with an N-terminal Flag tag. The following primer sequences were used to clone TRIP13: Forward, 5'-ACTATCTCGAGATGG ACGAGGCCGTGGGCGAC-3' and reverse, 5'-TCGATA GCGGCCGCTCAGATGTAAGCTGCAAGCTTC-3'. PCR was performed using PrimeSTAR Max DNA Polymerase (Takara Bio, Inc.). PCR was performed under the following conditions: Denaturation at $94^{\circ} \mathrm{C}$ for $10 \mathrm{sec}$, annealing at $55^{\circ} \mathrm{C}$ for $30 \mathrm{sec}$ and extension at $72^{\circ} \mathrm{C}$ for $1 \mathrm{~min}$. To generate a recombinant retrovirus, HEK293T cells were transfected with pQCXIP-Flag-TRIP13 together with pVPack-GP and pVPack-Ampho vectors (Promega Corporation) using Lipofectamine 2000 (Invitrogen; Thermo Fisher Scientific, Inc., Waltham, MA, USA). Following $48 \mathrm{~h}$, the supernatants were added to HCT116 or DLD1 cells in the presence of $2 \mu \mathrm{g} / \mathrm{ml}$ polybrene (Sigma-Aldrich; Merck Millipore, Darmstadt, Germany), and the infected cells were selected with $1 \mu \mathrm{g} / \mathrm{ml}$ puromycin for 3 days.

Immunoblot analysis. When the cells had reached $80 \%$ confluence, they were lysed using Laemmli sample buffer and boiled for $5 \mathrm{~min}$. The protein concentrations of the lysates were measured using the RC-DC Protein Assay (Bio-Rad Laboratories, Hercules, CA, USA). Equal protein quantities of the lysates were then separated on $0.01 \%$ SDS-PAGE gels and transferred to polyvinylidene fluoride membranes (Merck Millipore). Next, the membranes were blocked with $0.5 \%$ skim milk followed by incubation with anti-TRIP13 (1:1,000 dilution) and anti- $\beta$-actin antibody (1:3,000 dilution; cat. no. A1978; Sigma-Aldrich; Merck Millipore) primary antibodies for $1 \mathrm{~h}$ at room temperature. Subsequently, the membranes were incubated with horseradish peroxidase-conjugated secondary antibodies (1:3,000 dilution; cat. nos. 7074 and 7076; Cell Signaling Technology, Inc., Danvers, MA, USA) for $1 \mathrm{~h}$ at room temperature. Blots were visualized using Chemi-Lumi One Super (Nacalai Tesque, Inc., Kyoto, Japan).

Small interfering RNA (siRNA) transfection. The siRNA sequences used to deplete TRIP13 were 5'-GCUGGUAAC CAAGAUGUUUTT-3' (siTRIP13-1), 5'-CCCAUCGAUUUG AGUGCAUTT-3' (siTRIP13-2) and 5'-GGAUGCAUAAUG CCAGCAATT-3' (siTRIP13-3). The sequence of the control siRNA targeting luciferase was 5'-CUUACGCUGAGUACU UCGATT-3'. A total of $20 \mathrm{nM}$ of siRNAs were transfected into the cells using RNAiMAX (Invitrogen; Thermo Fisher Scientific, Inc.) according to the manufacturer's protocol. All siRNAs were purchased from Hokkaido System Science Co., Ltd. (Sapporo, Japan).

Cell invasion assay. To measure cell invasion using Boyden chambers, a filter was pre-coated with Matrigel, and $2 \times 10^{5}$ HCT116 or DLD1 cells transfected with the aforementioned siRNAs were seeded onto the upper surface of the chamber. Cells were fixed with $70 \%$ methanol $24 \mathrm{~h}$ later and stained with $0.5 \%$ crystal violet. The cells that had invaded the lower surface of the filters were counted in five randomly selected fields. Three independent experiments were performed, and the data were represented as the mean \pm standard deviation (SD).

Cell migration assay. Cell migration was evaluated using Boyden chambers. siRNA-transfected cells $\left(5.0 \times 10^{4}\right)$ were seeded onto the upper surface of the chamber. The lower surface of the filter was coated with fibronectin (Sigma-Aldrich; Merck Millipore). Cells were fixed with $70 \%$ methanol $6 \mathrm{~h}$ later and stained with $0.5 \%$ crystal violet. The cells that had migrated to the lower surface of the filters were counted in five randomly selected fields in three independent experiments.

Patients and ethics statement. CRC tissue samples and normal colorectal tissue sections were obtained from patients who had undergone surgery at Nagoya University Hospital (Nagoya, Japan) between May 2010 and October 2014. The study was approved by the institutional review board of Nagoya University Hospital and conformed to the standards set by the Declaration of Helsinki. All participants provided written informed consent to participate in the study.

Reverse transcription-quantitative PCR (RT-qPCR) analysis. RNA was extracted from CRC samples and cells using the RNeasy Mini kit (Qiagen GmbH, Hilden, Germany), and cDNA was generated using PrimeScript Reverse Transcriptase (Takara Bio, Inc.). The CRC tissue samples were obtained from patients at Nagoya University Hospital who had provided informed consent. qPCR was performed using the SYBR Premix Ex Taq ${ }^{\mathrm{TM}}$ II (Takara Bio, Inc.), and the Thermal Cycler Dice Real Time System TP800 (Takara Bio, Inc.) was used for the analysis. PCR was performed under the following conditions: $95^{\circ} \mathrm{C}$ for $10 \mathrm{sec}$ and $60^{\circ} \mathrm{C}$ for $30 \mathrm{sec}$. The relative messenger RNA (mRNA) expression levels were normalized to the level of glyceraldehyde 3-phosphate dehydrogenase (GAPDH) using LightCyclerR ${ }^{\circledR}$ Nano software 1.0 (Roche Diagnostics, Tokyo, Japan). The sequences of the primers used to amplify each gene were 5'-AGGTGGAGGAGTGGGTGT 
A

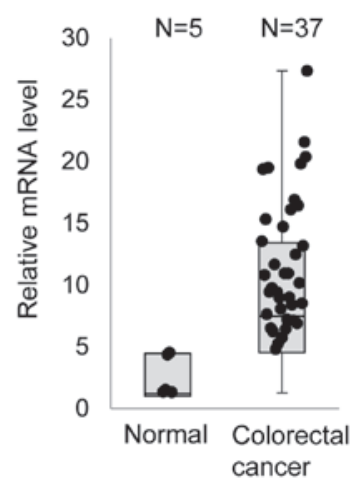

B

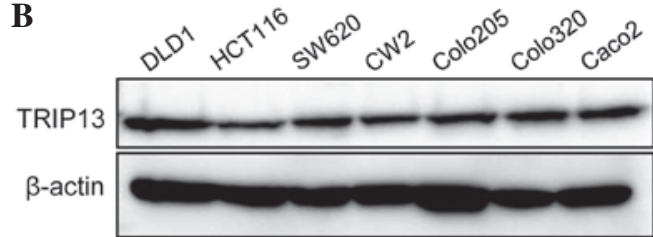

Figure 1. TRIP13 is expressed in CRC tissue specimens and cell lines. (A) The level of TRIP13 mRNA in CRC tissue specimens and normal colorectal tissues was evaluated by reverse transcription-quantitative polymerase chain reaction. The graph shows the relative level of TRIP13 mRNA normalized to the TRIP13 mRNA level in one of the normal samples. (B) The expression of TRIP13 in multiple CRC cell lines was examined by immunoblotting. mRNA, messenger RNA; TRIP13, thyroid hormone receptor interactor 13; CRC, colorectal cancer.

A

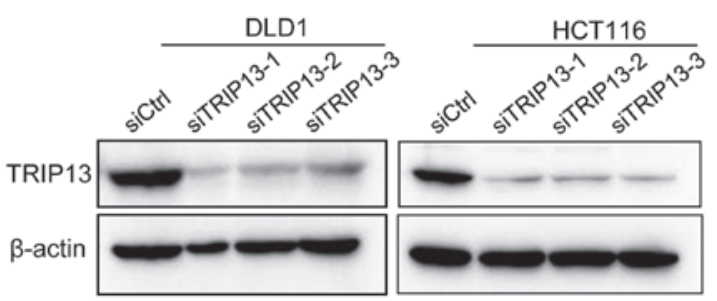

B
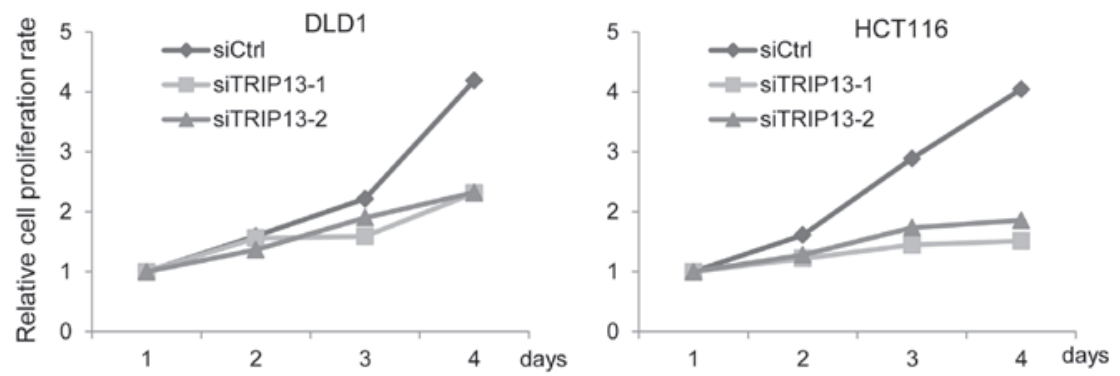

C

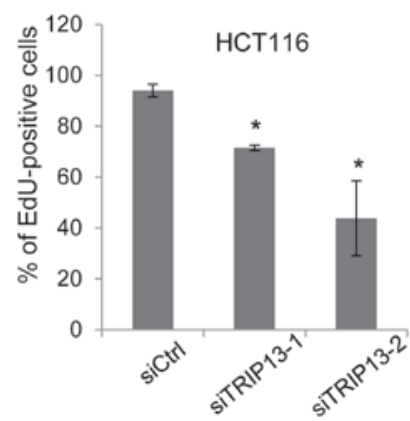

Figure 2. TRIP13 knockdown suppresses cell proliferation. (A) DLD1 or HCT116 cells were transfected with siRNAs, and $72 \mathrm{~h}$ later, the expression of TRIP13 was examined by immunoblotting. (B) Cells were transfected with siRNAs, and the number of viable cells at the indicated times was evaluated. (C) Cells were transfected with siRNAs and subjected to an EdU incorporation assay $48 \mathrm{~h}$ later. The graph shows the percentages of EdU-positive cells. Three independent experiments were performed, and the data are shown as the mean \pm standard deviation ("P<0.05). TRIP13, thyroid hormone receptor interactor 13; siRNA, small interfering RNA; Ctrl, control; EdU, 5-ethynyl-2'-deoxyuridine.

CGCTGTT-3' (forward) and 5'-CCGGGAAACTGTGGC GTGATGG-3' (reverse) for GAPDH, and 5'-CTGTCTCTG GCAGTGGACAAG-3' (forward) and 5'-TTGGTTTGCAGA AGGGATTC-3' (reverse) for TRIP13.
Cell proliferation assay. Cells were cultured in 96-well plates, and the number of viable cells at the indicated times was evaluated using Cell Counting Kit-8 (Dojindo Molecular Technologies, Inc., Kumamoto, Japan). 
A
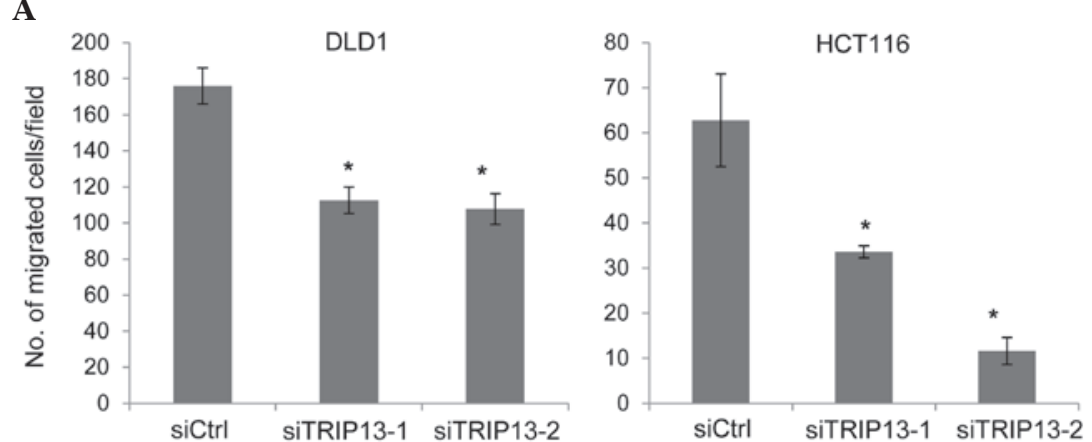

B
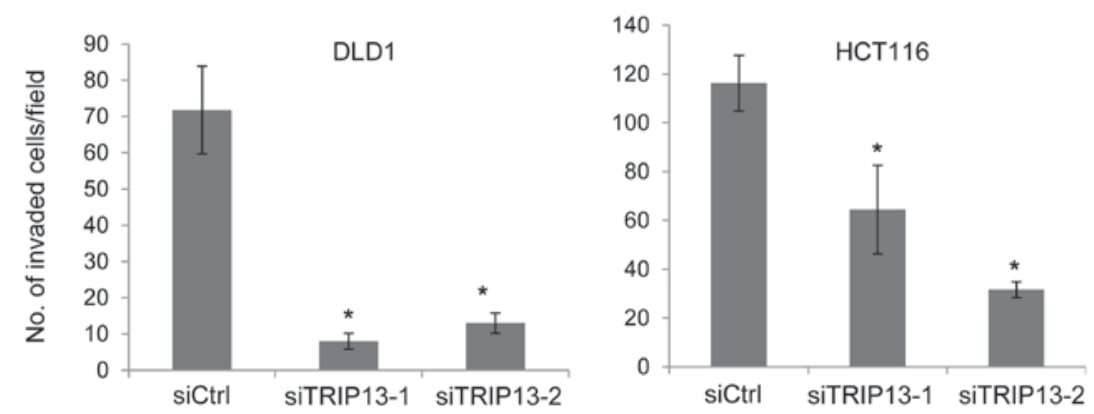

C

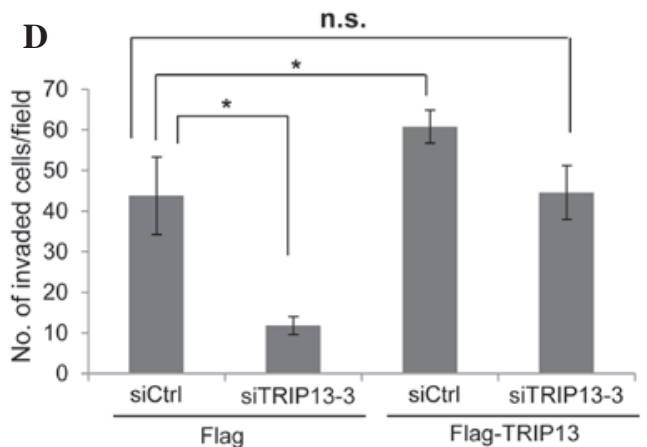

Figure 3. Knockdown of TRIP13 inhibits cell migration and invasion. (A) Cells were transfected with siRNAs, and $72 \mathrm{~h}$ later, cell migration was examined. The graph shows the average number of migrated cells per field. Three independent experiments were performed, and the data are shown as the mean \pm SD ( $\mathrm{P}<0.05)$. (B) siRNA-transfected cells were subjected to an invasion assay. The graph shows the average number of invaded cells per field. Three independent experiments were performed, and the data are shown as the mean \pm SD $($ P $<0.05)$. (C) HCT116 cells that constitutively expressed Flag tag or Flag-TRIP13 were established by retroviral infection. Cells were transfected with control siRNA or with TRIP13 siRNA (siTRIP13-3), and the expression of TRIP13 was examined by immunoblotting $72 \mathrm{~h}$ later. siTRIP13-3 targets the 3'-untranslated region of TRIP13 messenger RNA. The arrow indicates Flag-TRIP13 while the arrowhead indicates endogenous TRIP13. (D) Cells transfected with siRNAs were subjected to an invasion assay. The graph shows the average number of invaded cells per field. Three independent experiments were performed, and the data are shown as the mean \pm SD ( $\mathrm{P}<0.05)$. SD, standard deviation; TRIP13, thyroid hormone receptor interactor 13; siRNA, small interfering RNA; Ctrl, control; n.s., not significant.

5-ethynyl-2'-deoxyuridine (EdU) incorporation assay. CRC cells were transfected with siRNAs, and EdU incorporation assays were performed $48 \mathrm{~h}$ later using a Click-i ${ }^{\circledast}$ Plus EdU Alexa Fluor ${ }^{\circledR} 594$ Imaging kit (Thermo Fisher Scientific, Inc.). Briefly, siRNA-transfected cells were incubated with $20 \mathrm{mM}$ EdU for $24 \mathrm{~h}$ and fixed with formaldehyde. The cells were permeabilized with $0.5 \%$ Triton X-100 and stained with Hoechst in a reaction cocktail prepared according to the manufacturer's protocol. The cells were then imaged by fluorescence microscopy (BX60; Olympus Corporation, Tokyo, Japan), and the percentage of EdU-positive cells was evaluated.

Statistical analysis. Data were expressed as the mean \pm SD. Comparisons between groups were performed using unpaired $t$-tests. $\mathrm{P}<0.05$ was considered to indicate a statistically significant difference.

\section{Results}

TRIP13 is expressed in CRC tissues and cell lines. To examine whether TRIP13 has any role in CRC, the mRNA level of TRIP13 in CRC tissue specimens was first examined by RT-qPCR. As indicated in Fig. 1A, higher expression of TRIP13 was observed in multiple CRC samples compared with normal tissues. Next, the expression of TRIP13 in several CRC cell lines was examined. Immunoblot analyses demonstrated that all the cell lines expressed a similar amount of TRIP13 (Fig. 1B). 


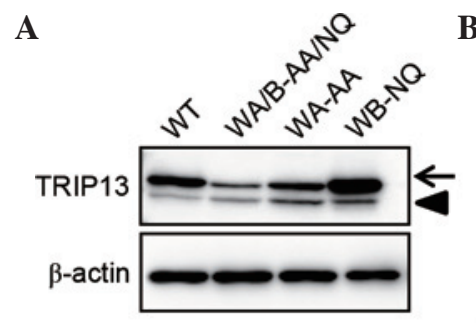

B
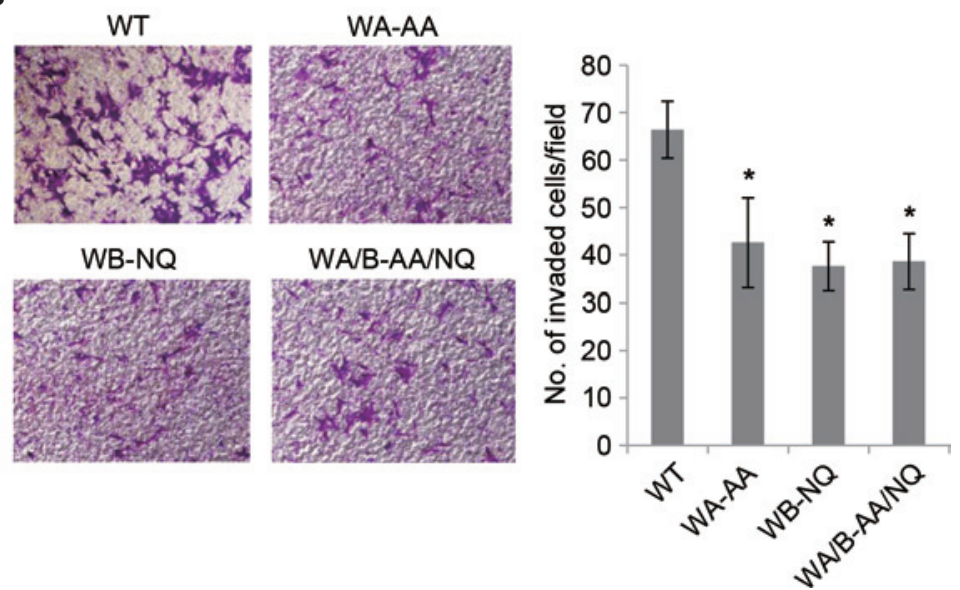

Figure 4. Expression of an inactive mutant of TRIP13 suppresses cell invasion. (A) HCT116 cells that constitutively expressed a Flag-tagged mutant TRIP13 were established by retrovirus infection. Cell lysates were subjected to immunoblotting with an anti-TRIP13 antibody. The arrow indicates Flag-tagged proteins and the arrowhead indicates endogenous TRIP13. (B) Cells were subjected to an invasion assay (stain, crystal violet; magnification, $\mathrm{x} 40$ ). Representative photographs are shown. The graph shows the average number of invaded cells per field. Three independent experiments were performed, and the data are shown as the mean \pm standard deviation $\left({ }^{*} \mathrm{P}<0.05\right)$. TRIP13, thyroid hormone receptor interactor 13; WT, wild type; WA, Walker A; WB, Walker B.

Depletion of TRIP13 suppresses cell proliferation. To determine whether TRIP13 has any tumor-promoting functions, TRIP13 expression was depleted in DLD1 and HCT116 cells using siRNAs that targeted different regions of TRIP13 mRNA. Transfection of either siRNA sufficiently knocked down TRIP13 expression (Fig. 2A). In the absence of TRIP13 expression, the proliferation of both cell lines was significantly suppressed (Fig. 2B). Reduced cell proliferation can be induced by a delay in cell cycle progression or by the promotion of cellular apoptosis (21). To determine how TRIP13 depletion suppressed cell proliferation, a terminal deoxynucleotidyl transferase dUTP nick end labeling (TUNEL) assay and an EdU incorporation assay were performed. The TUNEL assay detects fragmented DNA induced by apoptosis, while the EdU incorporation assay evaluates cell cycle progression (21). Cells that progress through the $\mathrm{S}$ phase incorporate the thymidine analog EdU; thus, proliferating cells can be detected based on the level of EdU incorporated (21). As shown in Fig. 2C, TRIP knockdown reduced the ratio of cells that incorporated EdU; however, no increase in the level of apoptosis caused by TRIP13 depletion was observed (data not shown).

TRIP13 knockdown inhibits cell migration and invasion. The effects of TRIP13 knockdown on cell migration were next studied using a modified Boyden chamber. DLD1 and HCT116 cells were transfected with siRNAs, and $72 \mathrm{~h}$ later, the cells were placed on the upper surface of the filter and allowed to migrate to the bottom surface, which was coated with fibronectin. The cells that migrated to the bottom surface were counted to evaluate cell migration. As shown in Fig. 3A, TRIP13 depletion significantly suppressed the migration of both cell lines. The invasion of TRIP13-knockdown cells was also examined using Matrigel-coated Boyden chambers. The invasion of both DLD1 and HCT116 cells was clearly suppressed by TRIP13 depletion (Fig. 3B).

To confirm that TRIP13 is associated with the invasion of cancer cells, a rescue experiment was performed.
HCT116 cells that constitutively expressed either Flag or Flag-TRIP13 were generated by retrovirus infection. To specifically deplete endogenous TRIP13, an additional siRNA (siTRIP13-3) that targeted the 3'-untranslated region of TRIP13 mRNA was used. Transfection of the siTRIP13-3 depleted endogenous TRIP13, but the expression level of Flag-TRIP13 was not affected by this siRNA (Fig. 3C). The invasion of siRNA-transfected cells was then examined using Matrigel-coated Boyden chambers. The invasion of Flag-expressing HCT116 cells was significantly reduced by transfection with siTRIP13-3 (Fig. 3D). Exogenous expression of Flag-TRIP13 significantly promoted cell invasion (Fig. 3D), while depletion of endogenous TRIP13 in Flag-TRIP13-expressing cells suppressed cell invasion to a level similar to that of control siRNA-transfected Flag-expressing cells (Fig. 3D). These results indicate that the reduction in cell invasion caused by the TRIP13 siRNAs was mediated by the depletion of TRIP13.

The catalytic activity of TRIP13 is required for the promotion of cell invasion. It was next examined whether the catalytic activity of TRIP13 was required for the promotion of cell invasion. Mutations in the Walker A motif are known to disrupt ATP binding, whereas Walker B motif mutations disrupt ATP hydrolysis (2). Both were shown to be essential for the function of TRIP13 in yeast (13). The present study generated TRIP13 mutants that have mutations either in the Walker A or Walker B motifs, or in both motifs, including TRIP13-GK/AA, which has glycine 184 and lysine 185 in the Walker A motif substituted with alanine; and TRIP13-DE/NQ, which has aspartic acid 252 and glutamic acid 253 in the Walker B motif substituted with asparagine and glutamine, respectively; and TRIP13-GK/AA-DE/NQ, which has both mutations. HCT116 cells that constitutively expressed Flag-tagged versions of each mutant were established by retrovirus infection. Although the expression of TRIP13-GK/AA and TRIP13-GK/AA-DE/NQ was lower than that of Flag-TRIP13, the expression level was higher than that of endogenous TRIP13 (Fig. 4A). The invasion 
of these cell lines was examined using Matrigel-coated Boyden chambers. As shown in Fig. 4B, the invasion of these mutant cell lines was significantly reduced compared with that of the wild type TRIP13-expressing cells. These results demonstrated that the catalytic activity of TRIP13 is required for the promotion of cell invasion.

\section{Discussion}

TRIP13 has been demonstrated to be a kinetochore-localized protein that ensures accurate progression of cell division (8-12). A number of kinetochore-localized proteins are highly expressed in various cancers, and their expression is associated with the genomic instability or malignant conversion of cancer cells (19). For example, aurora B, which is localized to the kinetochore (as well as to the spindle midzone) during mitosis, is a protein kinase critical for the accurate distribution of chromosomes (22). Aurora B is highly expressed in lung, prostate and thyroid cancers, and deregulated aurora B promotes tumorigenesis by inducing aneuploidy $(23,24)$. In addition to aurora B, other kinetochore proteins such as highly expressed in cancer 1 and monopolar spindle 1 kinase have also been shown to be overexpressed in certain cancers, and small molecules that inhibit the functions of these proteins are regarded as promising candidate agents for cancer treatment (25-27). These previous studies indicate the important functions of kinetochore-localized proteins for cancer progression. The present report revealed that TRIP13 was highly expressed in CRC tumor tissues and various CRC cell lines. High expression of TRIP13 has also been reported in head and neck cancer, as well as in prostate cancer $(20,28)$, indicating that TRIP13 may be overexpressed in a variety of cancers, similar to other kinetochore-localized proteins (25-27). Together with the current results, those studies suggest that TRIP13 is important in the progression of multiple cancers.

Mitotic defects often lead to aneuploidy, which promotes the genetic instability of cells and induces apoptosis or cellular transformation (19). The mitotic SAC ensures that all the kinetochores are properly attached to the mitotic spindle to mediate the accurate distribution of chromosomes (18). A critical component of the SAC is the mitotic checkpoint complex (MCC), which is composed of budding uninhibited by benzimidazole-related 1 , budding uninhibited by benzimidazoles 3 , mitotic arrest deficient (MAD) 2 and cell division cycle 20 (29,30). Deregulated expression or inactivation of MCC components is associated with numerous types of cancers. Mice with MAD2 or MAD1 heterozygous deletions were prone to develop lung cancer or various types of cancer, respectively $(31,32)$. The embryonic fibroblasts of these mice were defective at maintaining the SAC, and consequently exhibited a high frequency of aneuploidy (32). These results clearly demonstrate that maintenance of the SAC is critical to prevent cancer formation. TRIP13 has been shown to promote the dissociation of the MCC complex (15-17), which subsequently inactivates the SAC to prevent the completion of mitosis (18). Thus, overexpression of TRIP13 and silencing of MCC components have similar effects on cells, since both can lead to dysregulation of the SAC. Overexpression of TRIP13 may promote chromosomal instability in cancer cells, allowing further acquisition of malignant characteristics.
In summary, the present study has demonstrated that TRIP13 is overexpressed in CRC, and that the suppression of TRIP13 reduces CRC cell proliferation and invasion. TRIP13 has ATPase activity, and an inactivating mutant of TRIP13 was unable to promote cancer cell invasion, suggesting that the catalytic activity of TRIP13 is essential for cancer progression. Recent studies have reported that small chemical molecules can inhibit the activity of members of the AAA+ family (33). Thus, small chemicals that target TRIP13 may represent a promising drug for the treatment of various types of cancer.

\section{Acknowledgements}

The authors would like to thank the members of the Division of Cancer Biology, Nagoya University Graduate School of Medicine (Nagoya, Japan) for their helpful discussions and technical assistance. The present study was funded by a grant from The Naito Foundation (Tokyo, Japan).

\section{References}

1. Ogura T and Wilkinson AJ: AAA+ superfamily ATPases: Common structure-diverse function. Genes Cells 6: 575-597, 2001.

2. Hanson PI and Whiteheart SW: AAA+ proteins: Have engine, will work. Nat Rev Mol Cell Biol 6: 519-529, 2005.

3. Snider J and Houry WA: AAA+ proteins: Diversity in function, similarity in structure. Biochem Soc Trans 36: 72-77, 2008

4. Wendler P, Ciniawsky S, Kock M and Kube S: Structure and function of the AAA+ nucleotide binding pocket. Biochim Biophys Acta 1823: 2-14, 2012.

5. Grigoletto A, Lestienne P and Rosenbaum J: The multifaceted proteins Reptin and Pontin as major players in cancer. Biochim Biophys Acta 1815: 147-157, 2011.

6. Huber O, Ménard L, Haurie V, Nicou A, Taras D and Rosenbaum J: Pontin and reptin, two related ATPases with multiple roles in cancer. Cancer Res 68: 6873-6876, 2008.

7. Lee JW, Choi HS, Gyuris J, Brent R and Moore DD: Two classes of proteins dependent on either the presence or absence of thyroid hormone for interaction with the thyroid hormone receptor. Mol Endocrinol 9: 243-254, 1995.

8. Li XC and Schimenti JC: Mouse pachytene checkpoint 2 (trip13) is required for completing meiotic recombination but not synapsis. PLoS Genet 3: e130, 2007.

9. Roig I, Dowdle JA, Toth A, de Rooij DG, Jasin M and Keeney S: Mouse TRIP13/PCH2 is required for recombination and normal higher-order chromosome structure during meiosis. PLoS Genet 6: e1001062, 2010.

10. Ho HC and Burgess SM: Pch2 acts through Xrs2 and Tel1/ATM to modulate interhomolog bias and checkpoint function during meiosis. PLoS Genet 7: e1002351, 2011.

11. Wojtasz L, Daniel K, Roig I, Bolcun-Filas E, Xu H, Boonsanay V, Eckmann CR, Cooke HJ, Jasin M, Keeney S, et al: Mouse HORMAD1 and HORMAD2, two conserved meiotic chromosomal proteins, are depleted from synapsed chromosome axes with the help of TRIP13 AAA-ATPase. PLoS Genet 5: e1000702, 2009.

12. Farmer S, Hong EJ, Leung WK, Argunhan B, Terentyev Y, Humphryes N, Toyoizumi $\mathrm{H}$ and Tsubouchi $\mathrm{H}$ : Budding yeast Pch2, a widely conserved meiotic protein, is involved in the initiation of meiotic recombination. PLoS One 7: e39724, 2012.

13. Chen C, Jomaa A, Ortega $\mathbf{J}$ and Alani EE: Pch2 is a hexameric ring ATPase that remodels the chromosome axis protein Hop1. Proc Natl Acad Sci USA 111: E44-E53, 2014.

14. Tipton AR, Wang K, Oladimeji P, Sufi S, Gu Z and Liu ST: Identification of novel mitosis regulators through data mining with human centromere/kinetochore proteins as group queries. BMC Cell Biol 13: 15, 2012.

15. Wang K, Sturt-Gillespie B, Hittle JC, Macdonald D, Chan GK, Yen TJ and Liu ST: Thyroid hormone receptor interacting protein 13 (TRIP13) AAA-ATPase is a novel mitotic checkpoint-silencing protein. J Biol Chem 289: 23928-23937, 2014. 
16. Eytan E, Wang K, Miniowitz-Shemtov S, Sitry-Shevah D, Kaisari S, Yen TJ, Liu ST and Hershko A: Disassembly of mitotic checkpoint complexes by the joint action of the AAA-ATPase TRIP13 and p31 (comet). Proc Natl Acad Sci USA 111: 12019-12024, 2014.

17. Ye Q, Rosenberg SC, Moeller A, Speir JA, Su TY and Corbett KD: TRIP13 is a protein-remodeling AAA+ ATPase that catalyzes MAD2 conformation switching. Elife 4: 2015.

18. Lara-Gonzalez P, Westhorpe FG and Taylor SS: The spindle assembly checkpoint. Curr Biol 22: R966-R980, 2012.

19. Rao CV, Yamada HY, Yao Y and Dai W: Enhanced genomic instabilities caused by deregulated microtubule dynamics and chromosome segregation: A perspective from genetic studies in mice. Carcinogenesis 30: 1469-1474, 2009.

20. Banerjee R, Russo N, Liu M, Basrur V, Bellile E, Palanisamy N, Scanlon CS, van Tubergen E, Inglehart RC, Metwally T, et al: TRIP13 promotes error-prone nonhomologous end joining and induces chemoresistance in head and neck cancer. Nat Commun 5: 4527, 2014.

21. Wong M, Hyodo T, Asano E, Funasaka K, Miyahara R, Hirooka Y, Goto H, Hamaguchi M and Senga T: Silencing of STRN4 suppresses the malignant characteristics of cancer cells. Cancer Sci 105: 1526-1532, 2014.

22. Giet R, Petretti C and Prigent C: Aurora kinases, aneuploidy and cancer, a coincidence or a real link? Trends Cell Biol 15 . 241-250, 2005.

23. Gautschi O, Heighway J, Mack PC, Purnell PR, Lara PN Jr and Gandara DR: Aurora kinases as anticancer drug targets. Clin Cancer Res 14: 1639-1648, 2008.

24. Nguyen HG, Makitalo M, Yang D, Chinnappan D, St Hilaire C and Ravid K: Deregulated Aurora-B induced tetraploidy promotes tumorigenesis. FASEB J 23: 2741-2748, 2009.
25. Huang LY, Chang CC, Lee YS, Chang JM, Huang JJ, Chuang SH, Kao KJ, Lau GM, Tsai PY, Liu CW, et al: Activity of a novel Hecl-targeted anticancer compound against breast cancer cell lines in vitro and in vivo. Mol Cancer Ther 13: 1419-1430, 2014.

26. Huang LY, Chang CC, Lee YS, Huang JJ, Chuang SH, Chang JM, Kao KJ, Lau GM, Tsai PY, Liu CW, et al: Inhibition of Hecl as a novel approach for treatment of primary liver cancer. Cancer Chemother Pharmacol 74: 511-520, 2014.

27. Maachani UB, Kramp T, Hanson R, Zhao S, Celiku O, Shankavaram U, Colombo R, Caplen NJ, Camphausen K and Tandle A: Targeting MPS1 enhances Radiosensitization of human Glioblastoma by modulating DNA repair proteins. Mol Cancer Res 13: 852-862, 2015.

28. Larkin SE, Holmes S, Cree IA, Walker T, Basketter V, Bickers B, Harris S, Garbis SD, Townsend PA and Aukim-Hastie C: Identification of markers of prostate cancer progression using candidate gene expression. Br J Cancer 106: 157-165, 2012.

29. Sudakin V, Chan GK and Yen TJ: Checkpoint inhibition of the APC/C in HeLa cells is mediated by a complex of BUBR1, BUB3, CDC20, and MAD2. J Cell Biol 154: 925-936, 2001.

30. Tipton AR, Tipton M, Yen T and Liu ST: Closed MAD2 (C-MAD2) is selectively incorporated into the mitotic checkpoint complex (MCC). Cell Cycle 10: 3740-3750, 2011.

31. Sotillo R, Schvartzman JM, Socci ND and Benezra R: Mad2-induced chromosome instability leads to lung tumour relapse after oncogene withdrawal. Nature 464: 436-440, 2010.

32. Iwanaga Y, Chi YH, Miyazato A, Sheleg S, Haller K, Peloponese JM Jr, Li Y, Ward JM, Benezra R and Jeang KT: Heterozygous deletion of mitotic arrest-deficient protein 1 (MAD1) increases the incidence of tumors in mice. Cancer Res 67: 160-166, 2007.

33. Chapman E, Maksim N, de la Cruz F and La Clair JJ: Inhibitors of the AAA+ chaperone p97. Molecules 20: 3027-3049, 2015. 